\title{
Characterising the shared genetic determinants of bipolar disorder, schizophrenia and risk-taking
}

\author{
Guy Hindley $\mathrm{ID}^{1,2,11^{凶}}$, Shahram Bahrami ${ }^{1,11}$, Nils Eiel Steen $\mathbb{D}^{1}{ }^{1}$, Kevin S. O'Connell $\mathbb{D}^{1}$, Oleksandr Frei ${ }^{1,3}$, Alexey Shadrin (DD $^{1}$, \\ Francesco Bettella (D) ${ }^{1}$, Linn Rødevand ${ }^{1}$, Chun C. Fan ${ }^{4,5}$, Anders M. Dale $\mathbb{D}^{4,6,7,8}$, Srdjan Djurovic (D) ${ }^{9,10}$, Olav B. Smeland (D) ${ }^{1}$ and \\ Ole A. Andreassen (iD)
}

(c) The Author(s) 2021

Increased risk-taking is a central component of bipolar disorder (BIP) and is implicated in schizophrenia (SCZ). Risky behaviours, including smoking and alcohol use, are overrepresented in both disorders and associated with poor health outcomes. Positive genetic correlations are reported but an improved understanding of the shared genetic architecture between risk phenotypes and psychiatric disorders may provide insights into underlying neurobiological mechanisms. We aimed to characterise the genetic overlap between risk phenotypes and SCZ, and BIP by estimating the total number of shared variants using the bivariate causal mixture model and identifying shared genomic loci using the conjunctional false discovery rate method. Summary statistics from genome wide association studies of SCZ, BIP, risk-taking and risky behaviours were acquired $(n=82,315-466,751)$. Genomic loci were functionally annotated using FUMA. Of $8.6-8.7 \mathrm{~K}$ variants predicted to influence BIP, $6.6 \mathrm{~K}$ and $7.4 \mathrm{~K}$ were predicted to influence risk-taking and risky behaviours, respectively. Similarly, of 10.2-10.3 K variants influencing SCZ, 9.6 and $8.8 \mathrm{~K}$ were predicted to influence risk-taking and risky behaviours, respectively. We identified 192 loci jointly associated with SCZ and risk phenotypes and 206 associated with BIP and risk phenotypes, of which 68 were common to both risk-taking and risky behaviours and 124 were novel to SCZ or BIP. Functional annotation implicated differential expression in multiple cortical and sub-cortical regions. In conclusion, we report extensive polygenic overlap between risk phenotypes and BIP and SCZ, identify specific loci contributing to this shared risk and highlight biologically plausible mechanisms that may underlie risk-taking in severe psychiatric disorders.

Translational Psychiatry (2021)11:466; https://doi.org/10.1038/s41398-021-01576-4

\section{INTRODUCTION}

Schizophrenia (SCZ) and bipolar disorder (BIP) are severe mental disorders with overlapping clinical characteristics that are leading causes of morbidity and mortality worldwide [1]. With cardiovascular disease and suicide prominent in both [2-4], a better understanding of risk-taking behaviours such as smoking [5], substance use [6] and self-harm [7], could improve health outcomes [8]. Investigating genetic and neurobiological processes underlying the relationship between risk-taking, risky behaviours and SCZ and BIP may therefore offer novel opportunities for riskstratification and intervention.

Risk-taking is defined as a willingness to engage in behaviours not only with potential reward but also potential harm [9]. Typified by certain risky behaviours such as overspending, it is a core feature of BIP and contributes to diagnostic criteria for the disorder $[10,11]$. While a pronounced increase in risk-taking is associated with manic episodes [12], abnormalities in impulsivity, risk aversion and risk-seeking behaviour are also present as trait markers in euthymic people with BIP [13-15]. Additionally, mood stabilisers and anti-psychotics reduce impulsive and aggressive behaviour across a range of diagnoses, implying a shared neurobiological process to risk-taking beyond BIP $[16,17]$.

Unlike BIP, pronounced changes in risk-taking is not a core clinical feature of SCZ. However risky behaviours such as substance use, smoking and violence are more prevalent in individuals with SCZ than the general population [18-20]. Violence, in particular, is strongly associated with impulsivity, a neuropsychological domain closely related to risk-taking [20]. Findings from self-reports and neuropsychological measures are mixed. In one study, SCZ was not associated with self-reported risk perception [21] but Reddy et al. reported that individuals with SCZ were more risk averse than both individuals with BIP and healthy controls in a behavioural task [22]. Moreover, impulsivity has been shown to be both increased and decreased in subjects with SCZ,

\footnotetext{
${ }^{1}$ NORMENT, Institute of Clinical Medicine, University of Oslo and Division of Mental Health and Addiction, Oslo University Hospital, 0407 Oslo, Norway. ${ }^{2}$ Psychosis Studies, Institute of Psychiatry, Psychology and Neurosciences, King's College London, London, UK. ${ }^{3}$ Center for Bioinformatics, Department of Informatics, University of Oslo, Blindern, 0316 Oslo, Norway. ${ }^{4}$ Department of Neurology, Division of Clinical Neuroscience, Oslo University Hospital, Oslo, Norway. ${ }^{5}$ Multimodal Imaging Laboratory, University of California

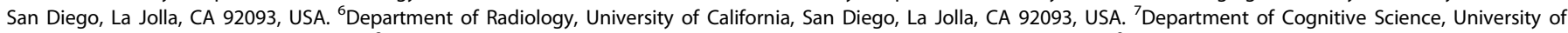
California, San Diego, La Jolla, CA, USA. ${ }^{8}$ Department of Psychiatry, University of California, San Diego, La Jolla, CA, USA. ${ }^{9}$ Department of Medical Genetics, Oslo University Hospital, Oslo, Norway. ${ }^{10}$ NORMENT, Department of Clinical Science, University of Bergen, Bergen, Norway. ${ }^{11}$ These authors contributed equally: Guy Hindley, Shahram Bahrami.

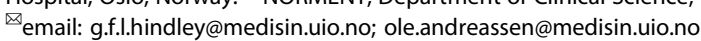


likely dependent on the subtype of SCZ and the presence or absence of psychosis [22, 23].

Various experimental studies have attempted to delineate the neurobiology underlying risk-taking, BIP and SCZ. Impulsivity and dysfunctional reward processing in BIP and SCZ have been associated with deficits in the prefrontal cortex on both functional and structural measures [23-26], loss of grey matter in the anterior cingulate cortex (ACC) $[27,28]$ and reduced white matter integrity in the cingulum and frontal lobes $[29,30]$. Moreover, dopaminergic neurotransmission in the mesolimbic reward system has been strongly implicated in risk-taking and risky behaviours [31], demonstrated by the association between impulse control disorders and dopamine agonists [32].

A recent genome-wide association study (GWAS) of risktaking and risky behaviours in over 1 million participants has provided new insights into their genetic architecture [33]. Using questionnaire measures for the propensity to take risks and the first principal component of four risky behaviours, 99 risk-taking loci were identified, 46 of which were shared with risky behaviours, implicating glutamatergic and GABAergic neurotransmission [33]. Significant positive genetic correlations were reported between risk-taking and BIP $\left(r_{\mathrm{g}}=0.21\right)$ and SCZ $\left(r_{\mathrm{g}}=\right.$ $0.17)$, suggesting a shared genetic basis $[33,34]$. However, little is known about the individual genetic loci driving these findings. Moreover, genetic correlation does not provide a complete representation of the shared genetic architecture between two phenotypes [35]. This is demonstrated by recent evidence of overlap between similar complex polygenic phenotypes with multiple shared loci, but a mixture of concordant and opposite effects leading to minimal genetic correlation [36, 37]. Additionally, both BIP and SCZ are highly heritable, with SNP-based heritability estimates ranging from 20 to $40 \%$ [38-46]. An improved understanding of this genetic component will provide insights into their aetiology, and identify novel targets for prevention and treatment [47].

We therefore employed the bivariate causal mixture model (MiXeR) [43] and the conjunctional false discovery rate method (conjFDR) [36, 48] to large-scale GWASs for BIP [38] and SCZ [40] together with risk-taking and risky behaviours [33] in order to a) quantify the total number of shared variants regardless of effect direction, b) identify individual loci driving the phenotypic overlap between risk, SCZ and BIP and c) leverage polygenic overlap to boost statistical power to identify novel loci associated with SCZ and BIP.

\section{METHODS \\ Samples}

We acquired GWAS summary statistics from recent publications. The SCZ sample comprised 35,476 cases with SCZ and 46,839 controls [40]. The BIP sample comprised 41,917 cases and 371,549 controls [49]. The risk-taking cohort comprised 466,751 individuals [33]. All samples were of European descent. Four lakhs thirty-one thousand one hundred and twenty-six of the risk-taking sample were derived from the UK Biobank (UKB), and were assessed by a single yes/no item asking, "would you describe yourself as someone who takes risks?" The remaining 35,445 comprised ten individual cohorts. Risky behaviours were measured by calculating the first principal component of four risky behaviours in UKB (automobile speeding propensity, alcoholic drinks per week, number of sexual partners and ever smoker") $(n=315,894)$. These items were chosen because they have been shown to correlate with self-reported risk-taking in independent samples [50-54], represent distinct domains of risk-taking (namely driving, alcohol drinking, smoking and sexual behaviours), and they were available in the entire sample [33]. For further details see supplementary methods and the original publications $[33,38,40]$. The Regional Committee for Medical Research Ethics-Southeast Norway has evaluated the current protocol, and found that no additional institutional review board approval was necessary as no individual data were used. Relevant ethics committees approved all primary GWASs, and all participants provided informed consent $[33,39,49]$.

\section{Data analysis}

We employed MiXeR to quantify polygenic overlap between each psychiatric disorder and risk phenotype [43]. A bivariate Gaussian mixture model using GWAS summary statistics was constructed to estimate the total number of shared and phenotype-specific variants that explains $90 \%$ of SNP heritability in each phenotype. Model fit is based on likelihood maximisation of signed test statistics (GWAS $z$-scores) evaluated by the Akaike Information Criterion (AIC), and demonstrated with predicted versus observed conditional quantile-quantile $(\mathrm{Q}-\mathrm{Q})$ plots. See supplementary methods and supplementary Fig. 1 for further information. We also calculated LD-score regression genetic correlation [42].

Conditional Q-Q plots were constructed to visualise cross-trait enrichment between each pair of phenotypes. Conditional Q-Q plots compare the association between individual SNPs and a primary phenotype (e.g., SCZ) as a function of their association with a secondary phenotype (e.g., risk-taking). Cross-trait enrichment is present if there are successive leftward deflections from the expected Q-Q plot under the null hypothesis (i.e., that there are no SNPs associated with the primary phenotype), signifying a higher proportion of SNPs associated with the primary phenotype as the strength of association with the secondary phenotype increases [48].

To identify individual SNPs jointly associated with both phenotypes, we employed conjFDR analysis using a threshold of conjFDR <0.05 [48]. Further details of the conjFDR analysis can be found in supplementary methods and prior publications [36, 48, 55, 56]. ConjFDR is also able to identify novel associations with each phenotype beyond genome-wide significance due to the boost in power from the cross-trait analysis.

\section{Genomic loci definition}

Independent significant SNPs, lead SNPs, candidate SNPs, and genomic loci margins were defined using the FUMA protocol (http://fuma.ctglab.nl/). See supplementary methods for further information. Novel loci were determined by cross-referencing identified loci with previous GWASs and other relevant studies [33, 37-40, 48, 55, 57-66].

\section{Functional annotation}

Putative causal genes were mapped to lead SNPs using three genemapping methodologies: 1) positional mapping which matches SNPs to their nearest genes, 2) expression quantitative trait loci (eQTL) mapping that identifies genes whose expression is associated with the SNPs' allelic variation, 3) chromatin interaction mapping that matches SNPs to genes with which they are predicted to interact based on chromatin structure [67]. We conducted differential gene expression analyses using GTEx eQTL data, Gene Ontology gene-set analyses using FUMA $[68,69]$, pathway analyses using Consensus PathDB [70] and spatiotemporal gene expression analysis of mapped genes using BrainSpan RNA sequencing data [71-73]. All analyses were corrected for multiple comparisons using Bonferroni correction. Further details are provided in Supplementary methods.

All code is publicly available at https://github.com/precimed.

\section{RESULTS}

\section{Estimating total genetic overlap}

MiXeR demonstrated substantial polygenic overlap between BIP, $\mathrm{SCZ}$, and each of risk-taking and risky behaviours, beyond that captured by genetic correlation (Fig. 1). Of a total of $8.6-7 \mathrm{~K}$ variants estimated to influence $\mathrm{BIP}, 6.6 \mathrm{~K}(\mathrm{SD}=2.0 \mathrm{~K}, 77 \%)$ and $7.4 \mathrm{~K}(\mathrm{SD}=0.7,85 \%)$ were also estimated to influence risk-taking and risky behaviours, respectively. Similarly, of a total of $10.2-10.3 \mathrm{~K}$ variants predicted to influence SCZ (N.B. estimates of polygenicity for individual traits can differ between analyses due to the random-pruning process), $9.6 \mathrm{~K}(\mathrm{SD}=0.5 \mathrm{~K}, 94 \%)$ and $8.8 \mathrm{~K}$ $(\mathrm{SD}=0.7,85 \%)$ were predicted to influence risk-taking and risky behaviours, respectively. We also found highly significant positive genetic correlations between all phenotypic pairings (risk-taking/ BIP: $r_{\mathrm{q}}=0.33, p=2.35 \mathrm{e}^{-31}$; risky behaviours/BIP: $r_{\mathrm{g}}=0.24, p=$ 7.50 ${ }^{-16}$; risk-taking/SCZ: $r_{\mathrm{g}}=0.22, p=7.41 \mathrm{e}^{-16}$; risky behaviours/ SCZ: $\left.r_{\mathrm{g}}=0.16, p=2.08 \mathrm{e}^{-10}\right)$, replicating previous findings $[8,33]$. Despite this, the extent of the overlap in relation to the size of the genetic correlations indicated a mixture of shared variants with concordant and discordant effects on each pair of phenotypes. Accordingly, MiXeR estimated that $57-72 \%$ (SD $0.5-12 \%$ ) of 


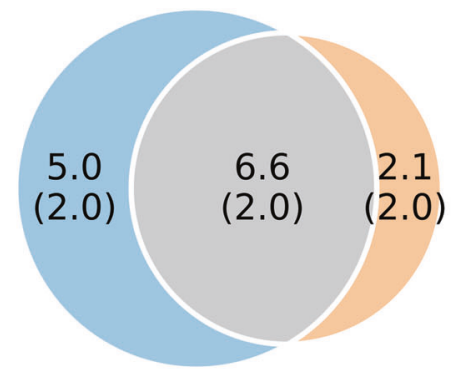

$r_{g}=0.33$

a. Risk-taking \& BIP
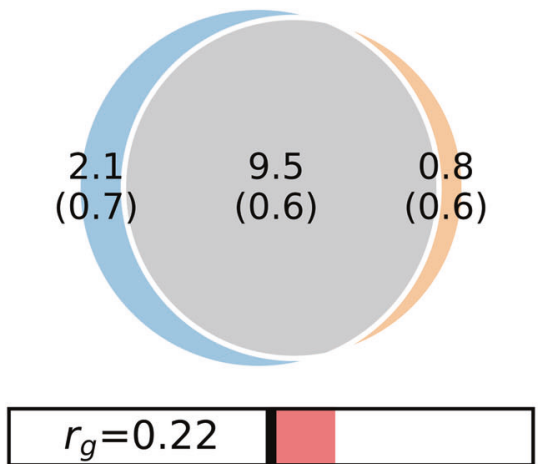

c. Risk-taking \& SCZ

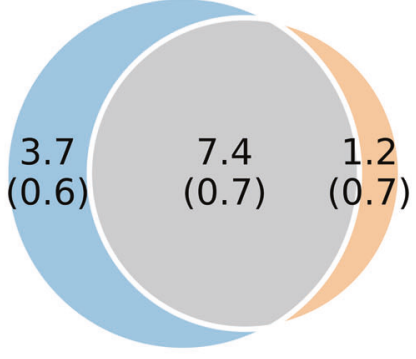

$r_{g}=0.24$

b. Risky behaviours \& BIP

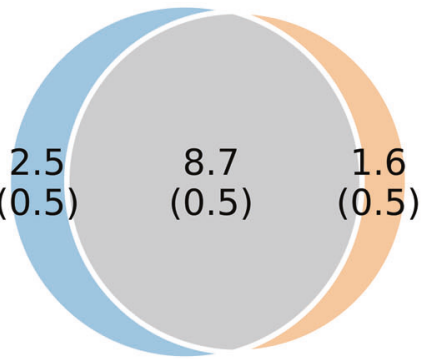

$r_{g}=0.16$

d. Risky behaviours \& SCZ

Fig. 1 Genome-wide genetic overlap between risk phenotypes, bipolar disorder and schizophrenia. MiXeR analysis for a bipolar disorder (BIP) and risk-taking, b BIP and risky behaviours, c schizophrenia (SCZ) and risk-taking, and d SCZ and risky behaviours. Venn diagrams representing the unique and shared variants associated with risk-taking and risky behaviours and each of SCZ and BIP. Polygenic overlap is represented in grey, Risk/risky behaviours in blue and BIP/SCZ in orange. The numbers indicate the estimated quantity of variants in thousands per component that explains $90 \%$ of SNP heritability for each phenotype (standard error in parentheses). The size of the circle reflects the extent of polygenicity for each trait.

shared SNPs had concordant effects (Supplementary Table 1). MiXeR also illustrated all four phenotypes' extensive polygenicity. Risk-taking and risky behaviours were estimated to be particularly polygenic $(11.5 \mathrm{~K}$ and $11.1 \mathrm{~K}$ variants respectively), helping to explain why smaller proportions of risk-taking and risky behaviourassociated variants were predicted to influence mental disorders (57-83\%). Model fit was adequate (further details in the Supplementary results).

\section{Visualising cross-trait enrichment}

Conditional Q-Q plots demonstrated step-wise increments in SNP enrichment for SCZ and BIP as a function of the strength of their association with risk-taking and risky behaviours (Fig. 2), and for risk-taking and risky behaviours as a function of their association with SCZ and BIP (Supplementary Fig. 2). This further demonstrated cross-trait enrichment between phenotypes.

\section{Shared loci between SCZ, BIP and risk-taking}

At a threshold of conjFDR $<0.05$, we identified 106 and 131 loci jointly associated with BIP and each of risk-taking and risky behaviours respectively, 98 of which were novel in BIP and 31 were overlapping across both risk phenotypes (Fig. 3, Table 1, and Supplementary Table 2). 88\% (93/106) and 85\% (111/131) shared the same direction of effect on BIP and risk-taking and risky behaviours, respectively, in line with the positive genetic and MiXeR predictions. With regards SCZ, there were 100 and 129 loci jointly associated with SCZ and each of risk-taking and risky behaviours, respectively, of which 38 were novel in SCZ (Fig. 3 and Supplementary Table 3). Furthermore, 37 loci were overlapping across both risk-taking and risky behaviours analyses (Table 2), and 18 were also overlapping with loci associated with BIP and both risk phenotypes (Table 1 and Supplementary Tables 2-3). $76 \%(76 / 100)$ and $74 \%(96 / 129)$ of all lead SNPs had the same direction of effect on SCZ and each of risk-taking and risky behaviours, respectively.

\section{Functional annotation}

We mapped 142 and 177 protein-coding genes to lead SNPs for BIP and each of risk-taking and risky behaviours respectively (Supplementary Tables 4 and 5). Thirty-nine genes were mapped to both sets of lead SNPs, including the calcium channel CACNA1C and the synaptic cell adhesion molecule CADM2 [74]. Expression of mapped genes was significantly enriched in 30 and 25 tissues for risk-taking and risky behaviours, respectively (Supplementary Figs. 3 and 4). The amygdala, hippocampus, anterior cingulate and multiple basal ganglia structures were among the top ten tissues for both analyses. Gene-set analysis identified 50 gene-sets significantly enriched with mapped genes for risk-taking and BIP, 14 of which were specific to neuronal structure (Supplementary Table 6) and pathway analysis identified 19 overrepresented pathways (Supplementary Table 7). Regarding risky behaviours and bipolar, 36 gene-sets were enriched, 14 of which were also enriched in risk-taking and bipolar, including seven of the neuronal structure gene-sets. Thirty-seven pathways were over- 

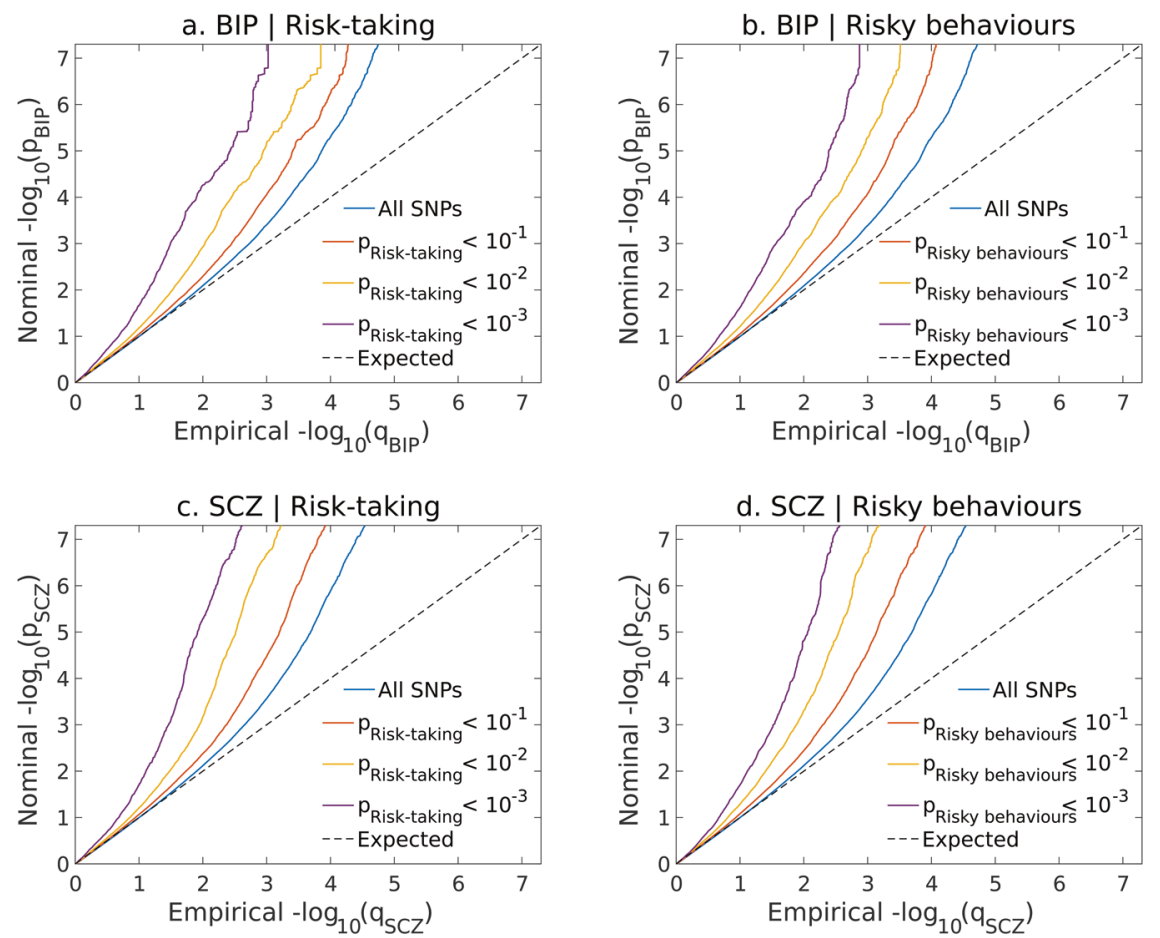

Fig. 2 Polygenic enrichment of SNPs associated with bipolar disorder (BIP) and schizophrenia (SCZ) dependent on their association with risk taking and risky behaviours. Visualising cross-trait enrichment between risk phenotypes, bipolar disorder and schizophrenia. Conditional Q-Q plots of nominal versus empirical $-\log 10 p$ values in $\operatorname{BIP}(\mathbf{a}, \mathbf{b})$ and $\operatorname{SCZ}(\mathbf{c}, \mathbf{d})$ above the threshold of $p<5 \times 10^{-8}$ as a function of the significance of their association with risk-taking (a./c.) and risky behaviours (RiskyBehav) (b./d.) at the level of $p<0.1, p<0.01$ and $p<0.001$, respectively.

represented with mapped genes for risky behaviours and BIP, five of which were associated with GABA-ergic neurotransmission. Further, "twelve loci associated with ADHD", "protein-protein interactions at synapse" and "nicotine addiction" were also present in BIP and risk-taking (Supplementary Tables 6 and 7).

With regards SCZ, we mapped a total of 131 and 181 genes to lead SNPs associated with SCZ and each of risk-taking and risky behaviours, respectively (supplementary Tables 8 and 9). Twentynine were mapped to lead SNPs from both analyses, including the GABA receptor subunit GABRA2 [75] and EPHA5, a tyrosine kinase implicated in neurodevelopment (Table 2) [76]. When testing differential tissue expression of mapped genes, three structures in the basal ganglia (caudate, putamen and nucleus accumbens) were the most significantly enriched tissues for both analyses (Supplementary Figs. 5 and 6). Gene-set analysis identified 34 gene-sets enriched with mapped genes for risk-taking and $\mathrm{SCZ}$, and 54 for risky behaviours and SCZ (Supplementary Table 10). Among these, 19 were common to both risk phenotypes, 14 of which were related to neuron development, structure or function. Pathway analysis of the same sample of genes identified 18 and 30 pathways significantly overrepresented with mapped genes for SCZ and each of risk-taking and risky behaviours, respectively. A single pathway, "Twelve loci atssociated with ADHD", was common to all four analyses (Supplementary Table 11). Further functional annotations and spatiotemporal gene expression analyses are presented in Supplementary results, Supplementary Tables 12-15, and Supplementary Figs. 7 and 8.

\section{DISCUSSION}

In this analysis of GWAS summary statistics, we reveal extensive polygenic overlap between mental disorders and risk phenotypes beyond genetic correlation and identify and characterise independent genomic loci underlying this overlap. Using MiXeR, we first estimated that $77-94 \%$ of all BIP or SCZ influencing variants also influence risk-taking and risky behaviours, despite moderate positive genetic correlations. This has implications for how the genetic risk for mental-health-related traits is conceptualised, suggesting most variants influence multiple traits with few phenotype-specific variants. We next identified 206 genomic loci jointly associated with BIP and risk phenotypes and 192 associated with SCZ and risk phenotypes using conjFDR. Of these, 98 were novel in BIP and 38 were novel in SCZ, contributing to ongoing efforts to reveal the missing heritability of SCZ and BIP. Furthermore, $74-88 \%$ of lead SNPs had concordant effects on mental disorders and risk phenotypes, in line with positive genetic correlations. Finally, we highlight the role of multiple cortical and sub-cortical brain structures and neuronal development, structure, and function in risk phenotypes and both disorders. These findings may lead to the new mechanistic hypotheses, the identification of novel treatment targets and enable risk stratification of risk-taking and risky behaviours in severe psychiatric disorders.

Using MiXeR, we demonstrated that most variants associated with BIP and SCZ also influence risk-taking and risky behaviours, despite genetic correlations between 0.16-0.30 [43]. While this may be surprising, genetic correlation provides a summary measure between -1 and 1 of the correlation of effect sizes. This means that mixtures of variants with concordant and opposite effects "cancel each other out", resulting in a genetic correlation of 0 . The extensive overlap is therefore compatible with these modest genetic correlation estimates since MiXeR predicted $57-72 \%$ of shared variants had concordant effect sizes. While genetic correlation is useful to understand how the overall genetic risk for one phenotype covaries with the genetic risk for another, uncovering the fraction of overlapping and unique variants provides another dimension to the characterisation of shared genetic architecture. Indeed, these findings are consistent with a growing body of evidence suggesting that, despite differing genetic correlations, there is widespread polygenic overlap of a 

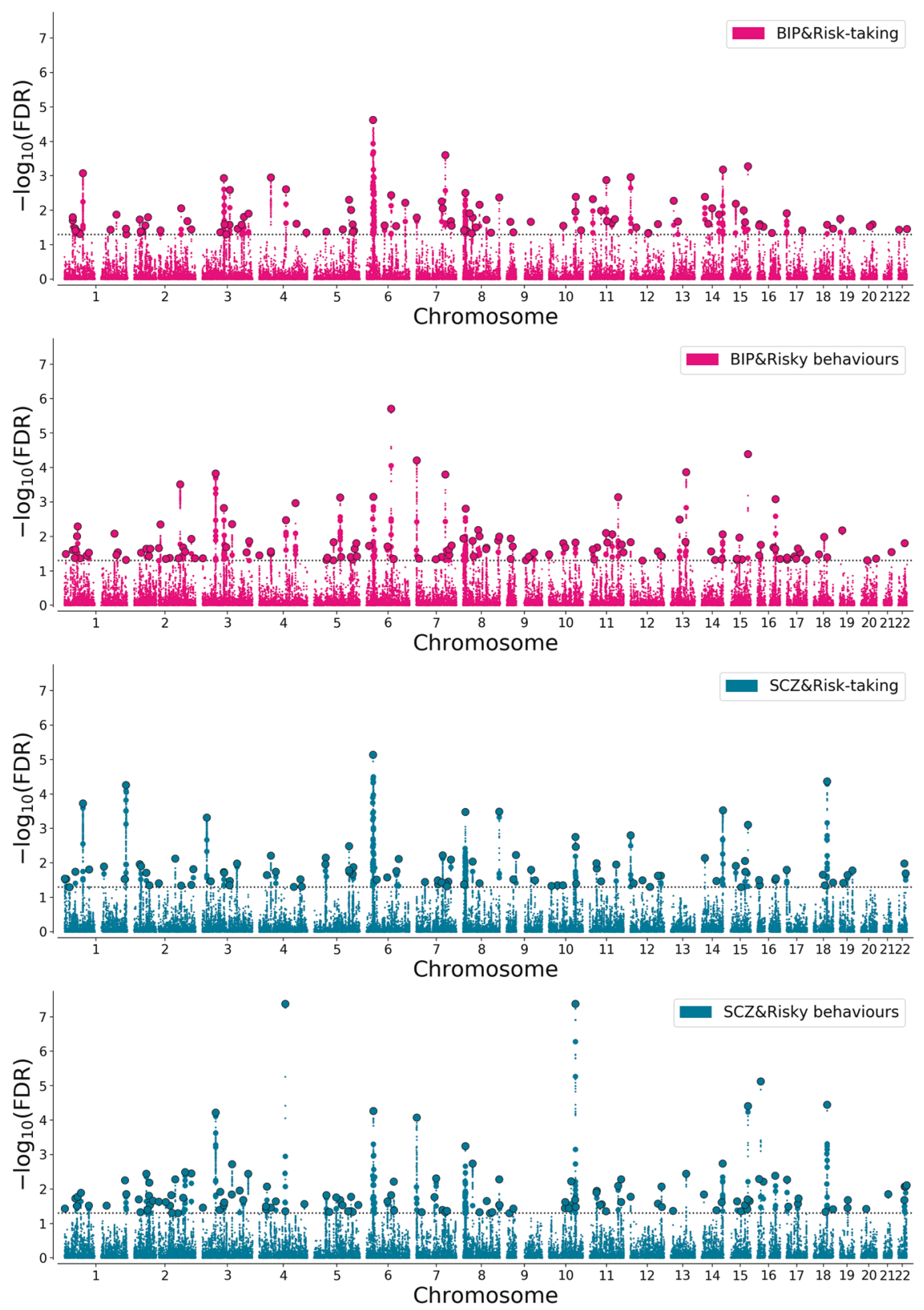

Fig. 3 Discovery of genetic loci jointly associated with risk phenotypes, bipolar disorder and schizophrenia. Manhattan plots showing the $-\log 10$ transformed conjFDR values for A bipolar disorder (BIP) and risk-taking, B BIP and risky behaviours, C schizophrenia (SCZ) and risktaking and D SCZ and risky behaviours risk for each SNP (y-axis) against chromosomal position ( $x$-axis). The dotted line represents the threshold for significant association (conjFDR < 0.05). Black circles represent lead SNPs.

similar extent across a diverse range of mental-health-related phenotypes, including almost total overlap between SCZ and educational attainment $[35,77]$. Taken together, these findings have implications for how the genetic risk of complex polygenic traits, like BIP and SCZ, is conceptualised. If polygenic overlap is the norm, then each risk variant is likely to be highly non-specific and influence multiple diverse traits. This would imply that it is, in fact, the specific distribution of effect sizes and effect directions, along with the interaction between different risk variants, that differentiates risk for a specific phenotype rather than a specific set of variants [35].

Nonetheless, the higher proportion of concordant lead SNPs and moderate positive genetic correlations indicate a genetic basis to the increased risk-taking and risky behaviours observed in BIP, suggesting that risk-taking may represent a genetically influenced endophenotype for BIP [15]. In contrast, risk-taking has been reported to be both increased and decreased in SCZ [22], while risky behaviours, such as smoking and violence, are increased. Our findings therefore suggest that there is a similar genetic tendency for risk-taking and risky behaviours in $\mathrm{SCZ}$, as with BIP. This indicates that conflicting findings in SCZ are likely to be influenced by methodological and environmental factors, such as the use of neuropsychiatric measures that correlate poorly with self-report measures [78], antipsychotic medication use [21] and cognitive symptoms [79] rather than differences in genetic influences. This also suggests that differences in risk-taking and risk-behaviour may be a trait-marker in SCZ as in BIP, although this requires further investigation [13-15].

We next used conjFDR to identify specific genetic loci jointly associated with each mental disorder and risk phenotype. 
Table 1. Ten genomic loci jointly associated with bipolar disorder (BIP) and risk-taking which physically overlapped with loci jointly associated with BIP and risky behaviours at conjunctional FDR (conjFDR) $<0.05$.

\begin{tabular}{|c|c|c|c|c|c|c|c|}
\hline Chr & Min-max BP & Lead SNPs & ConjFDR & $\begin{array}{l}\text { Direction } \\
\text { of effect }\end{array}$ & Novel in BIP & $\begin{array}{l}\text { Novel in } \\
\text { risk-taking }\end{array}$ & Mapped genes \\
\hline 1 & $44,029,353-44,196,945$ & rs2367724 & 0.039 & + & $x$ & Yes & $M E D 8^{\mathrm{b}}, K D M 4 A^{\mathrm{b}}, A R T N^{\mathrm{b}}$ \\
\hline 1 & $205,646,278-205,799,987$ & rs823130 & 0.013 & + & $x$ & $x$ & $\begin{array}{l}\text { NUCKS } S^{\mathrm{b}}, R A B 7 L 1^{\mathrm{b}}, S L C 41 A 1^{\mathrm{b}}, \\
\text { PM20D1 } 1^{\mathrm{b}}\end{array}$ \\
\hline 1 & $243,503,764-243,579,112$ & rs4146671 & 0.034 & + & Yes & $x$ & $S D C C A G 8^{\mathrm{b}}$ \\
\hline 2 & $225,334,070-225,467,840$ & rs2047134 & 0.036 & + & Yes & $x$ & CUL $3^{\mathrm{b}}$ \\
\hline 3 & $84,841,679-85,789,644$ & rs9831123 ${ }^{a}$ & 0.001 & + & $x$ & $x$ & $C A D M 2^{\mathrm{b}}$ \\
\hline 3 & $94,027,330-94,229,614$ & rs4857445 & 0.050 & + & $x$ & Yes & - \\
\hline 4 & $46,191,375-46,361,545$ & rs535066 & 0.001 & + & Yes & $x$ & $G A B R A 2^{\mathrm{b}}$ \\
\hline 4 & $106,061,534-106,417,856$ & $r s 2647256^{a}$ & 0.002 & + & $x$ & $x$ & $T E T 2^{\mathrm{b}}$ \\
\hline 6 & $98,310,291-98,792,109$ & rs1487445 & 0.004 & + & $x$ & $x$ & - \\
\hline 7 & $1,860,733-2,110,850$ & rs11768212 & 0.016 & + & $x$ & Yes & $M A D 1 L 1^{\mathrm{b}}$ \\
\hline 7 & $114,940,147-115,117,353$ & rs10251192 & 0.000 & + & Yes & $x$ & - \\
\hline 7 & $140,116,033-140,182,514$ & rs80274100 & 0.028 & + & $x$ & Yes & $R A B 19^{\mathrm{b}}, M K R N 1^{\mathrm{b}}$ \\
\hline 8 & $9,306,087-10,006,664$ & rs73207111 & 0.003 & + & $x$ & $x$ & - \\
\hline 8 & $38,014,429-38,310,910$ & rs11777067 & 0.016 & + & $\mathrm{x}$ & Yes & $L S M 1^{\mathrm{b}}$ \\
\hline 8 & $65,437,506-65,499,486$ & rs7813444 ${ }^{\mathrm{a}}$ & 0.007 & + & Yes & $x$ & $\begin{array}{l}\text { DDHD2 } 2^{\mathrm{b}}, P P A P D C 1 B^{\mathrm{b}}, W H S C 1 L 1^{\mathrm{b}} \\
F G F R 1^{\mathrm{b}}\end{array}$ \\
\hline 8 & $92,976,563-93,180,965$ & rs28716374 & 0.019 & + & $x$ & Yes & - \\
\hline 8 & $143,363,229-143,404,118$ & rs34853464 & 0.004 & + & $x$ & Yes & $R U N X 1 T 1^{\mathrm{b}}$ \\
\hline 15 & $33,761,014-33,878,554$ & rs2071382 & 0.001 & + & $x$ & Yes & FURIN ${ }^{\mathrm{b}}$, FES $^{\mathrm{b}}$ \\
\hline 16 & $44,029,353-44,196,945$ & rs6500948 & 0.028 & + & Yes & Yes & RBFOX $1^{\mathrm{b}}$ \\
\hline
\end{tabular}

Chr chromosome, min-max BP minimum-maximum base pair position, ConjFDR conjFDR value of lead SNP, Direction of effect -"+" concordant effect directions of lead SNPs, "+/-" discordant effect direction of lead SNPs on BIP and risk-taking, Novel BIP/risk-taking: "Yes" indicates novel loci, Mapped genes genes mapped to lead SNPs for BIP and risk-taking.

a'Lead SNPs that were also lead SNPs in the risky behaviour loci. For corresponding BIP and risky behaviour loci please refer to Supplementary Table 3.

${ }^{\mathrm{b}}$ Genes that were also mapped to lead SNPs for BIP and risky behaviours.

'This locus is within the major histocompatibility complex. Given the complex LD in this region, gene mapping strategies are not reliable. Mapped genes are provided but should be interpreted with caution.

Through leveraging the cross-trait enrichment to boost statistical power, this enabled the identification of 98 novel risk loci in BIP and 38 in SCZ, Although these findings require further validation $[36,47]$. A more complete understanding of the genomic architecture of SCZ and BIP is necessary to aid the translation of genetic research into clinical practice through more accurate polygenic risk scores and better defined neurobiological targets $[80,81]$. We also identified 68 loci that were common to risk-taking and risky behaviours, thus increasing the validity of these findings. This approach was also utilised in the original risk-taking GWAS given the limitations of using single-item questionnaire measures [33].
We functionally annotated all jointly associated loci to explore putative biological mechanisms linking the polygenic overlap and phenotypic associations observed between risk-taking, BIP and SCZ. GABAergic pathways were implicated in both disorders via the several GABA-related gene-sets for BIP and risky behaviours, and $G A B R(A)$ gene linked to $S C Z$ and both risk phenotypes, in line with findings in the original risk-taking GWAS [33]. It is also notable that CACNA1C was mapped to lead SNPs from all four analyses, while CADM2 was mapped to lead SNPs from BIP and both risk-taking and risky behaviours. Interestingly, both genes were also implicated in a recent GWAS of impulsivity and drug experimentation [74]. Additionally, lead SNPs in the shared loci 
Table 2. Thirty-seven genomic loci jointly associated with schizophrenia (SCZ) and risk-taking which physical overlapped with loci jointly associated with SCZ and risky behaviours at conjunctional FDR (conjFDR) $<0.05$.

\begin{tabular}{|c|c|c|c|c|c|c|c|}
\hline Chr & Min-max BP & Lead SNPs & ConjFDR & $\begin{array}{l}\text { Direction } \\
\text { of effect }\end{array}$ & Novel in SCZ & $\begin{array}{l}\text { Novel in risk- } \\
\text { taking }\end{array}$ & Mapped genes \\
\hline 1 & $43,982,527-44,145,130$ & rs673253 & 0.018 & + & $x$ & Yes & $\begin{array}{l}\text { TMEM125, TIE1, MED8 }{ }^{\mathrm{b}} \text {, } \\
\text { PTPRF }^{\mathrm{b}}\end{array}$ \\
\hline 1 & $243,281,617-243,645,203$ & rs 10803138 & $<0.001$ & + & $x$ & $x$ & SDCCAG8 \\
\hline 2 & $48,176,656-48,707,841$ & rs138659652 & 0.019 & + & Yes & Yes & MSH6, FBXO11 \\
\hline 2 & $97,593,614-98,332,858$ & rs115507803 & 0.038 & + & $x$ & Yes & $F A H D 2 B$ \\
\hline 2 & $225,334,070-225,467,840$ & rs2047134 & 0.043 & + & $x$ & $\times$ & CUL3 ${ }^{\mathrm{b}}$ \\
\hline 3 & $84,841,679-84,961,810$ & rs1598080 & 0.018 & + & $x$ & $x$ & - \\
\hline 3 & $86,011,109-86,192,846$ & rs58783194 & 0.019 & $+1-$ & $x$ & Yes & - \\
\hline 4 & $31,162,143-31,201,229$ & rs7669969 $9^{a}$ & 0.022 & + & Yes & $x$ & - \\
\hline 4 & $46,191,375-46,361,545$ & rs10805144 & 0.006 & + & Yes & $x$ & $G A B R A 2^{\mathrm{b}}$ \\
\hline 7 & $114,940,147-115,113,279$ & rs $4275159^{a}$ & 0.039 & + & Yes & $x$ & - \\
\hline 7 & $121,952,981-122,008,804$ & rs $988720^{\mathrm{a}}$ & 0.048 & + & $x$ & Yes & $F E Z F 1^{\mathrm{b}}, C A D P S 2^{\mathrm{b}}$ \\
\hline $8^{c}$ & $8,088,230-12,203,305$ & rs9329221 & $<0.001$ & + & $x$ & $x$ & $M S R A^{\mathrm{b}}, F A M 167 A$ \\
\hline 8 & $38,014,429-38,310,910$ & rs7845911 & 0.009 & + & $x$ & Yes & $\begin{array}{l}\angle S M 1^{\mathrm{b}}, B A G 4^{\mathrm{b}}, D D H D 2^{\mathrm{b}}, \\
\text { PPAPDC1B }^{\mathrm{b}}\end{array}$ \\
\hline 8 & $65,437,964-65,498,165$ & rs6996198 & 0.039 & + & $x$ & $x$ & - \\
\hline 8 & $143,276,606-143,404,118$ & rs13281016 & $<0.001$ & + & $x$ & Yes & TSNARE $^{\mathrm{b}}$ \\
\hline 9 & $26,447,292-27,111,268$ & rs56409537 & 0.030 & + & $x$ & Yes & - \\
\hline 10 & $104,546,183-105,165,256$ & rs12416687 & 0.002 & $+1-$ & $x$ & Yes & $\begin{array}{l}A R L 3^{\mathrm{b}}, W B P 1 L^{\mathrm{b}}, B O R C S 7^{\mathrm{b}}, \\
A S 3 M T^{\mathrm{b}}\end{array}$ \\
\hline 10 & $106,417,957-106,560,225$ & rs12761679 & 0.003 & + & $x$ & Yes & SORCS $3^{\mathrm{b}}$ \\
\hline 15 & $63,460,009-63,547,859$ & rs6494397 & 0.050 & $+/-$ & Yes & Yes & $R P S 27 L$ \\
\hline 15 & $78,714,561-78,926,726$ & rs12442456 & 0.009 & $+/-$ & $x$ & Yes & IREB2, ADAMTST ${ }^{\mathrm{b}}$ \\
\hline 15 & $91,403,674-91,443,059$ & rs2071382 & 0.001 & + & $x$ & Yes & $F E S^{\mathrm{b}}$, FURIN $^{\mathrm{b}}$ \\
\hline 16 & $7,324,960-7,417,203$ & rs8046401 & 0.031 & + & $x$ & Yes & $R B F O X 1^{\mathrm{b}}$ \\
\hline 16 & $71,355,142-71,396,661$ & rs3826248 & 0.031 & + & $x$ & $x$ & $C M T R 2, D H O D H, D H X 38^{\mathrm{b}}$ \\
\hline 18 & $53,183,396-53,477,999$ & rs1382119 & $<0.001$ & + & $x$ & $x$ & - \\
\hline 19 & $30,982,165-31,052,954$ & rs10421376 & 0.022 & $+/-$ & $x$ & Yes & ZNF536 \\
\hline 22 & $41,485,593-42,396,890$ & rs75843224 & 0.010 & $+1-$ & $x$ & Yes & EP300 \\
\hline
\end{tabular}

Chr chromosome, min-max BP minimum-maximum base pair position, ConjFDR conjFDR value of lead SNP, Direction of effect " + " concordant effect directions of lead SNPs, "+/-" discordant effect direction of lead SNPs on SCZ and risk-taking, Novel SCZ/risk-taking: "Yes" indicates novel loci; Mapped genes-genes mapped to lead SNPs for SCZ and risk-taking.

aLead SNPs that were also lead SNPs in the risky behaviour loci. For corresponding SCZ and risky behaviour loci please refer to Supplementary Table 2.

${ }^{\mathrm{b}}$ Genes that were also mapped to lead SNPs for SCZ and risky behaviours.

${ }^{c}$ These loci reside within regions possessing complex LD structure and so gene mapping strategies are not reliable. Mapped genes are provided but should be interpreted with caution.

from all four analyses were significantly associated with altered gene expression in the caudate nucleus, nucleus accumbens, putamen, amygdala and hippocampus, anterior cingulate cortex and frontal cortex, among others. The finding of significant differential expression in the basal ganglia is particularly interesting given evidence of increased functional connectivity in the nucleus accumbens and increased striatal activity on taskbased fMRI in risk-taking adolescents $[82,83]$. More broadly, these regions mirror neuroimaging and electro-encephalogram studies which implicate the frontal cortex, the anterior cingulate cortex 
and the striatum in risk-taking in healthy volunteers [84, 85], SCZ $[26,30]$ and BIP [24]. Interestingly, with the addition of the amygdala and the hippocampus, these structures make up the frontal-striatal reward system circuitry [86]. Taken with experimental evidence linking dysfunctional reward system processing and risk-taking in BIP [87] and SCZ [88], this offers a plausible neurobiological mechanism underlying the SNP associations reported.

Our study had several limitations. Firstly, MiXeR analysis was not sufficiently powered to accurately quantify the shared and unique components beyond maximum possible overlap. Larger samples are required to provide more precise estimates, which would enable comparison of the size of overlap between phenotypes. Secondly, this analysis was using European samples only. It is essential that more diverse samples and improved methods for transancestral analysis are developed to widen the applicability of genetic studies. Thirdly, the single yes/no item used to measure risk-taking in the UKB cohort has limited reliability and construct validity and has rarely been used in the context of SCZ and BIP. In particular, it is possible that responses to this item were confounded by concurrent affective symptoms, concurrent substance use and history of mental health diagnosis. Nonetheless, the prevalence of BIP, SCZ and depressive symptoms were low in the risk-taking sample, minimising the effect of these potential confounders, this questionnaire measure correlates highly with a variety of distinct risk-related behaviours [50-54], and the simplicity of the item enabled the collection of a substantial sample size. We also focused our discussion on findings replicated across both risk phenotypes, which are likely to represent more valid findings. Fifthly, the risky behaviours phenotype was constructed using four distinct phenotypes, two of which were related to substance use. In addition to risk-taking, addictive behaviours also correlate with disinhibited personality types, conduct disorder and attention-deficit hyperactivity disorder, all three of which are in turn linked to the latent factor "externalising". It is therefore important to note that the phenotypic and genetic correlation between risk-taking and risky behaviour may be interpreted as a correlation between two related constructs rather than capturing the same underlying risk-taking construct. Finally, functional annotation of highly polymorphic genetic loci may be unstable. We therefore conducted several functional analyses using independent datasets, including FUMA, ConsensusPathDB and BrainSpan to triangulate these findings.

In summary, our findings reveal extensive polygenic overlap between risk phenotypes and mental disorders with implications for how the polygenic architecture of complex disorders are conceptualised. We also identify specific loci underpinning this overlap, including 38 novel SCZ loci, 98 novel BIP loci and 68 loci common to both risk phenotypes. Functional annotation offered insights into neurobiological mechanisms underpinning the phenotypic overlap between BIP, SCZ and risk-taking, highlighting convergent roles for GABAergic systems, neuronal structure and function and structures implicated in the fronto-striatal reward system. Future work is required to better delineate the molecular genetic mechanisms underlying these statistical associations, and determine their interaction with other psychiatric disorders.

\section{REFERENCES}

1. Ferrari AJ, Stockings E, Khoo JP, Erskine HE, Degenhardt L, Vos T, et al. The prevalence and burden of bipolar disorder: findings from the Global Burden of Disease Study 2013. Bipolar Disord. 2016. https://doi.org/10.1111/bdi.12423.

2. Pompili M, Gonda X, Serafini G, Innamorati M, Sher L, Amore M. et al. Epidemiology of suicide in bipolar disorders: a systematic review of the literature. Bipolar Disord. 2013;15:457-90.

3. Brugnoli R, Novick D, Haro JM, Rossi A, Bortolomasi M, Frediani S, et al. Risk factors for suicide behaviors in the observational schizophrenia outpatient health outcomes (SOHO) study. BMC Psychiatry. 2012;12:1.
4. DE Hert M, Correll CU, Bobes J, Cetkovich-Bakmas M, Cohen D, Asai I, et al. Physical illness in patients with severe mental disorders. I. Prevalence, impact of medications and disparities in health care. World Psychiatry. 2011;10:52-77.

5. Brailovskaia J, Schillack H, Assion HJ, Horn H, Margraf J. Risk-taking propensity and (un)healthy behavior in Germany. Drug Alcohol Depend. 2018;192:324-8.

6. Rieser NM, Shaul L, Blankers M, Koeter M, Schippers GM, Goudriaan AE. The predictive value of impulsivity and risk-taking measures for substance use in substance dependent offenders. Front Behav Neurosci. 2019;13:192

7. Müller A, Claes L, Smits D, Brähler E, De Zwaan M. Prevalence and correlates of self-harm in the German general population. PLoS One. 2016;11:e0157928.

8. Strawbridge RJ, Ward J, Cullen B, Tunbridge EM, Hartz S, Bierut L, et al. Genomewide analysis of self-reported risk-taking behaviour and cross-disorder genetic correlations in the UK Biobank cohort. Transl Psychiatry. 2018;8:1-11.

9. Leigh BC. Peril, chance, adventure: Concepts of risk, alcohol use and risky behavior in young adults. Addiction. 1999. https://doi.org/10.1046/j.13600443.1999.9433717.x.

10. American Psychiatric Association. Diagnostic and statistical manual of mental disorders (DSM-5 ${ }^{\circledR}$ ). Washington: American Psychiatric Publishing; 2013.

11. World Health Organization. The ICD-10 classification of mental and behavioural disorders: diagnostic criteria for research. Geneva: WHO; 1993.

12. Meade CS, Fitzmaurice GM, Sanchez AK, Griffin ML, McDonald LJ, Weiss RD. The relationship of manic episodes and drug abuse to sexual risk behavior in patients with co-occurring bipolar and substance use disorders: a 15-month prospective analysis. AIDS Behav. 2011;15:1829-33.

13. Najt P, Perez J, Sanches M, Peluso MA, Glahn D, Soares JC. Impulsivity and bipolar disorder. Eur Neuropsychopharmacol. 2007;17:313-20.

14. Chandler RA, Wakeley J, Goodwin GM, Rogers RD. Altered risk-aversion and riskseeking behavior in bipolar disorder. Biol Psychiatry. 2009;66:840-6.

15. Hıdıroğlu C, Demirci Esen Ö, Tunca Z, Neslihan Gürz Yalçìn S, Lombardo L, Glahn DC, et al. Can risk-taking be an endophenotype for bipolar disorder? A study on patients with bipolar disorder type I and their first-degree relatives. J Int Neuropsychol Soc. 2013;19:474-82.

16. Jones RM, Arlidge J, Gillham R, Reagu S, van den Bree M, Taylor PJ. Efficacy of mood stabilisers in the treatment of impulsive or repetitive aggression: Systematic review and meta-analysis. Br J Psychiatry. 2011;198:93-8.

17. van Schalkwyk Gl, Beyer C, Johnson J, Deal M, Bloch MH. Antipsychotics for aggression in adults: a meta-analysis. Prog Neuropsychopharmacol Biol Psychiatry. 2018;81:452-8.

18. Swartz MS, Wagner HR, Swanson JW, Stroup TS, McEvoy JP, Canive JM, et al. Substance use in persons with schizophrenia: baseline prevalence and correlates from the NIMH CATIE study. J Nerv Ment Dis. 2006;194:164-72.

19. de Leon J, Diaz FJ. A meta-analysis of worldwide studies demonstrates an association between schizophrenia and tobacco smoking behaviors. Schizophr Res. 2005;76:135-57.

20. Rund BR. A review of factors associated with severe violence in schizophrenia. Nord J Psychiatry. 2018;72:561-71.

21. Fischer BA, McMahon RP, Kelly DL, Wehring HJ, Meyer WA, Feldman S, et al. Risktaking in schizophrenia and controls with and without cannabis dependence. Schizophr Res. 2015;161:471-7.

22. Reddy LF, Lee J, Davis MC, Altshuler L, Glahn DC, Miklowitz DJ, et al. Impulsivity and risk taking in bipolar disorder and schizophrenia. Neuropsychopharmacology 2014;39:456-63.

23. Kaladjian A, Jeanningros R, Azorin J-M, Anton J-L, Mazzola-Pomietto P. Impulsivity and neural correlates of response inhibition in schizophrenia. Psychol Med. 2011;41:291-9.

24. Mason L, O'sullivan N, Montaldi D, Bentall RP, El-Deredy W. Decision-making and trait impulsivity in bipolar disorder are associated with reduced prefrontal regulation of striatal reward valuation. Brain 2014;137:2346-55.

25. Lee J, Choi S, Kang J, Won E, Tae WS, Lee MS, et al. Structural characteristics of the brain reward circuit regions in patients with bipolar I disorder: a voxel-based morphometric study. Psychiatry Res Neuroimaging. 2017;269:82-9.

26. Avsar KB, Weller RE, Cox JE, Reid MA, White DM, Lahti AC. An fMRI investigation of delay discounting in patients with schizophrenia. Brain Behav. 2013;3:384-401.

27. Schiffer B, Müller BW, Scherbaum N, Forsting M, Wiltfang J, Leygraf N, et al. Impulsivity-related brain volume deficits in schizophrenia-addiction comorbidity. Brain 2010;133:3093-103.

28. Tu PC, Kuan YH, Li CT, Su TP. Structural correlates of trait impulsivity in patients with bipolar disorder and healthy controls: a surface-based morphometry study. Psychol Med. 2017;47:1292-9.

29. Hoptman MJ, Ardekani BA, Butler PD, Nierenberg J, Javitt DC, Lim KO. DTI and impulsivity in schizophrenia: a first voxelwise correlational analysis. Neuroreport 2004;15:2467-70.

30. Scholz V, Houenou J, Kollmann B, Duclap D, Poupon C, Wessa M. Dysfunctional decision-making related to white matter alterations in bipolar i disorder. J Affect Disord. 2016;194:72-9. 
31. Sugam JA, Carelli RM. Rolling the dice: the importance of mesolimbic dopamine signaling in risky decision making. Neuropsychopharmacology 2013;38:248.

32. Voon V, Gao J, Brezing C, Symmonds M, Ekanayake V, Fernandez H, et al. Dopamine agonists and risk: impulse control disorders in Parkinson's disease. Brain 2011;134:1438-46.

33. Karlsson Linnér $R$, Biroli $P$, Kong $E$, Meddens $S$, Wedow $R$, Fontana $M A$, et al Genome-wide association analyses of risk tolerance and risky behaviors in over 1 million individuals identify hundreds of loci and shared genetic influences. Nat Genet. 2019;51:245-57.

34. Strawbridge RJ, Ward J, Lyall LM, Tunbridge EM, Cullen B, Graham N, et al. Genetics of self-reported risk-taking behaviour, trans-ethnic consistency and relevance to brain gene expression. Transl Psychiatry. 2018;8:1-11.

35. Smeland $O B$, Frei $O$, Dale $A M$, Andreassen $O A$. The polygenic architecture of schizophrenia-rethinking pathogenesis and nosology. Nat Res. 2020;16:366-79.

36. Smeland OB, Frei O, Shadrin A, O'Connell K, Fan CC, Bahrami S. et al. Discovery of shared genomic loci using the conditional false discovery rate approach. Hum Genet. 2019:139:1-10.

37. Smeland OB, Bahrami S, Frei O, Shadrin A, O'Connell K, Savage J, et al. Genomewide analysis reveals extensive genetic overlap between schizophrenia, bipolar disorder, and intelligence. Mol Psychiatry. 2019;25:844-53.

38. Stahl EA, Breen G, Forstner AJ, McQuillin A, Ripke S, Trubetskoy V, et al. Genomewide association study identifies 30 loci associated with bipolar disorder. Nat Genet. 2019;51:793-803.

39. Pardiñas AF, Holmans P, Pocklington AJ, Escott-Price V, Ripke S, Carrera N, et al Common schizophrenia alleles are enriched in mutation-intolerant genes and in regions under strong background selection. Nat Genet. 2018;50:381-9.

40. Psychiatric SWG of the et al. Bakphrenia-associated genetic loci. Nature. 2014;511:421-7.

41. Lee SH, DeCandia TR, Ripke S, Yang J, et al. Estimating the proportion of variation in susceptibility to schizophrenia captured by common SNPs. Nat Genet. 2012;44:247-50.

42. Bulik-Sullivan BK, Loh PR, Finucane HK, Ripke S, Yang J, et al. LD Score regression distinguishes confounding from polygenicity in genome-wide association studies. Nat Genet. 2015;47:291-5.

43. Frei O, Holland D, Smeland OB, Shadrin AA, Fan CC, Maeland S, et al. Bivariate causal mixture model quantifies polygenic overlap between complex traits beyond genetic correlation. Nat Commun. 2019;10:1-11.

44. Cross-Disorder Group of the Psychiatric Genomics C, Lee SH, Ripke S, Neale BM, Faraone SV, Purcell SM, et al. Genetic relationship between five psychiatric disorders estimated from genome-wide SNPs. Nat Genet. 2013;45:984-94.

45. Girard SL, Xiong L, Dion PA, Rouleau GA. Where are the missing pieces of the schizophrenia genetics puzzle? Curr Opin Genet Dev. 2011;21:310-6.

46. Gershon ES, Alliey-Rodriguez N, Liu C. After GWAS: searching for genetic risk for schizophrenia and bipolar disorder. Am J Psychiatry. 2011;168:253-6.

47. Visscher PM, Wray NR, Zhang Q, Sklar P, McCarthy MI, Brown MA, et al. 10 Years of GWAS discovery: biology, function, and translation. Am J Hum Genet. 2017;101:5-22.

48. Andreassen OA, Djurovic S, Thompson WK, Schork AJ, Kendler KS, O'Donovan MC et al. Improved detection of common variants associated with schizophrenia by leveraging pleiotropy with cardiovascular-disease risk factors. Am J Hum Genet. 2013;92:197-209.

49. Mullins N, Forstner AJ, O'Connell KS, Coombes B, Coleman J, Qiao Z, et al. Genome-wide association study of more than 40,000 bipolar disorder cases provides new insights into the underlying biology. Nat Genet. 2021. https://doi. org/10.1038/s41588-021-00857-4.

50. Becker A, Deckers T, Dohmen T, Falk A, Kosse F. The relationship between economic preferences and psychological personality measures. Annu Rev Econ 2012;4:453-78.

51. Falk A, Becker A, Dohmen TJ, Enke B, Huffman D, Sunde U. The nature and predictive power of preferences: global evidence. SSRN Electronic Journal. 2015. https://doi.org/10.2139/ssrn.2691910.

52. Dohmen T, Falk A, Huffman D, Sunde U, Schupp J, Wagner GG. Individual risk attitudes: measurement, determinants, and behavioral consequences. J Eur Econ Assoc. 2011;9:522-50.

53. Beauchamp JP, Cesarini D, Johannesson M. The psychometric and empirical properties of measures of risk preferences. J Risk Uncertain. 2017;54:203-37.

54. Barsky RB, Juster FT, Kimball MS, Shapiro MD. Preference parameters and behavioral heterogeneity: an experimental approach in the health and retirement study. Q J Econ. 1997;112:537-79.

55. Andreassen OA, Thompson WK, Schork AJ, Ripke S, Mattingsdal M, Kelsoe JR, et al. Improved detection of common variants associated with schizophrenia and bipolar disorder using pleiotropy-informed conditional false discovery rate. PLoS Genet. 2013:9:e1003455.

56. Smeland OB, Wang Y, Frei O, Li W, Hibar DP, Franke B, et al. Genetic overlap between schizophrenia and volumes of hippocampus, putamen, and intracranial volume indicates shared molecular genetic mechanisms. Schizophr Bull. 2018;44:854-64.

57. Smeland OB, Shadrin A, Bahrami S, Broce I, Tesli M, Frei O. et al. Genome-wide association analysis of Parkinsonas disease and schizophrenia reveals shared genetic architecture and identifies novel risk loci. Biol Psychiatry. 2020;89:227-35.

58. Bahrami S, Steen NE, Shadrin A, O'Connell K, Frei O, Bettella F. et al. Shared genetic loci between body mass index and major psychiatric disorders: a genome-wide association study. JAMA Psychiatry. 2020;77:503-12.

59. Lam M, Chen CY, Li Z, Martin AR, Bryois J, Ma X, et al. Comparative genetic architectures of schizophrenia in East Asian and European populations. Nat Genet. 2019;51:1670-8.

60. Le Hellard S, Wang Y, Witoelar A, Zuber V, Bettella F, Hugdahl K, et al. Identification of gene loci that overlap between schizophrenia and educational attainment. Schizophr Bull. 2017;43:654-64.

61. O'connell KS, Shadrin A, Bahrami S, Smeland OB, Bettella F, Frei O, et al. Identification of genetic overlap and novel risk loci for attention-deficit/hyperactivity disorder and bipolar disorder. Mol Psychiatry. 2019. https://doi.org/10.1038/ s41380-019-0613-z

62. Lee PH, et al. Genomic relationships, novel loci, and pleiotropic mechanisms across eight psychiatric disorders. Cell 2019;179:1469-82.

63. Mullins $N$ et al. Genome-wide association study of over 40,000 bipolar disorder cases provides novel biological insights. MedRxiv. 2020. https://doi.org/10.1101/ 2020.09.17.2018705.

64. Drange OK, Smeland OB, Shadrin AA, Finseth PI, Witoelar A, Frei O, et al. Genetic overlap between Alzheimer's disease and bipolar disorder implicates the MARK2 and VAC14 genes. Front Neurosci. 2019;13:220.

65. Andreassen OA, Thompson WK, Dale AM. Genetic pleiotropy between multiple sclerosis and schizophrenia but not bipolar disorder: differential involvement of immune-related gene loci. Mol Psychiatry. 2015;20:207-14.

66. van der Meer D, Rokicki J, Kaufmann T, Córdova-Palomera A, Moberget T, Alnæs $D$, et al. Brain scans from 21,297 individuals reveal the genetic architecture of hippocampal subfield volumes. Mol Psychiatry. 2020;25:3053-65.

67. Shannon P, Markiel A, Ozier O, Baliga NS, Wang JT, Ramage D, et al. Cytoscape: a software environment for integrated models of biomolecular interaction networks. Genome Res. 2003;13:2498-504.

68. Watanabe K, Taskesen E, van Bochoven A, Posthuma D. Functional mapping and annotation of genetic associations with FUMA. Nat Commun. 2017;8:1826.

69. Boyle AP, Hong EL, Hariharan M, Cheng $Y$, Schaub MA, Kasowski $M$, et al. Annotation of functional variation in personal genomes using RegulomeDB. Genome Res. 2012;22:1790-7.

70. Herwig R, Hardt $C$, Lienhard M, Kamburov A. Analyzing and interpreting genome data at the network level with ConsensusPathDB. Nat Protoc. 2016;11:1889-907.

71. Miller JA, Ding SL, Sunkin SM, Smith KA, Ng L, Szafer A, et al. Transcriptional landscape of the prenatal human brain. Nature 2014;508:199-206.

72. BrainSpan. BrainSpan Atlas of the Developing Human Brain. 2010. http://www. brainspan.org/. Accessed 16 October 2020

73. Bahl E, Koomar T, Michaelson JJ. cerebroViz: an R package for anatomical visualization of spatiotemporal brain data. Bioinformatics 2017;33:762-3.

74. Sanchez-Roige S, Fontanillas P, Elson SL, Gray JC, de Wit H, Mackillop J, et al. Genome-wide association studies of impulsive personality traits (BIS-11 and UPPS-P) and drug experimentation in up to 22,861 adult research participants identify loci in the CACNA1I and CADM2 genes. J Neurosci. 2019;39:2562-72.

75. Gonzalez-Nunez V. Role of gabra2, GABA(A) receptor alpha-2 subunit, in CNS development. Biochem Biophys Rep. 2015;3:190-201.

76. Wilkinson DG. Multiple roles of eph receptors and ephrins in neural development. Nat Rev Neurosci. 2001;2:155-64.

77. Bahrami S. et al. Genetic loci shared between major depression and intelligence with mixed directions of effect. Nat Hum Behav. 2021;5:1-7.

78. Lejuez CW, Aklin WM, Zvolensky MJ, Pedulla CM. Evaluation of the balloon analogue risk task (BART) as a predictor of adolescent real-world risk-taking behaviours. J Adolesc. 2003;26:475-9.

79. Pedersen A, Göder R, Tomczyk S, Ohrmann P. Risky decision-making under risk in schizophrenia: a deliberate choice? J Behav Ther Exp Psychiatry. 2017;56:57-64.

80. Torkamani A, Wineinger NE, Topol EJ. The personal and clinical utility of poly genic risk scores. Nat Rev Genet. 2018;19:581-90.

81. Lally J, MacCabe JH. Personalised approaches to pharmacotherapy for schizophrenia. BJPsych Adv. 2016;22:78-86.

82. DeWitt SJ, Aslan S, Filbey FM. Adolescent risk-taking and resting state functional connectivity. Psychiatry Res Neuroimaging. 2014;222:157-64.

83. Chein JM, Albert D, O'Brien L, Uckert K, Steinberg L. Peers increase adolescent risk taking by enhancing activity in the brain's reward circuitry. Dev Sci. 2011;14: F1-10.

84. Chase HW, Fournier JC, Bertocci MA, Greenberg T, Aslam H, Stiffler R, et al. A pathway linking reward circuitry, impulsive sensation-seeking and risky decision- 
making in young adults: identifying neural markers for new interventions. Transl Psychiatry. 2017;7:e1096-e1096.

85. Schneider S, Peters J, Bromberg U, Brassen S, Miedl SF, Banaschewski T, et al. Risk taking and the adolescent reward system: a potential common link to substance abuse. Am J Psychiatry. 2012;169:39-46.

86. Nestler EJ, Hyman SE, Malenka RC. Molecular neuropharmacology: a foundation for clinical neuroscience. Pennsylvania: McGraw-Hill Medical; 2001.

87. Phillips ML, Swartz HA. A critical appraisal of neuroimaging studies of bipolar disorder: toward a new conceptualization of underlying neural circuitry and a road map for future research. Am J Psychiatry. 2014;171:829-43.

88. Strauss GP, Waltz JA, Gold JM. A review of reward processing and motivational impairment in schizophrenia. Schizophr Bull. 2014;40:S107-16.

\section{ACKNOWLEDGEMENTS}

We would like to thank the research participants and employees of the Working Groups of the Psychiatric Genomics Consortium and UK Biobank for access to data, and the Social Science Genetic Association Consortium for making available their GWAS summary statistics, and the many people who provided DNA samples. Biosamples and/or data for this publication were obtained from NIMH Repository \& Genomics Resource, a centralized national biorepository for genetic studies of psychiatric disorders. This research has been conducted using data from UK Biobank, a major biomedical database (Project ID number 27412; www.ukbiobank.ac.uk). The current study was supported by the American National Institutes of Health (NS057198, EB00790), the Research Council of Norway (223273, 248778, 262656, 273291), the South-Eastern Norway Regional Health Authority (2019-108, 2017-112), KG Jebsen Stiftelsen, the European Research Council under the European Union's Horizon 2020 research and Innovation program (Grant\# 802998). GH was supported by the European Union's Horizon2020 Research and Innovation Action Grant \# 847776 CoMorMent. SB was supported by the Norwegian Health Association (22731) and EU JPND: PMI-AD RCN 311993. The CommonMind Consortium was supported by funding from Takeda Pharmaceuticals Company Limited, F. Hoffmann-La Roche Ltd and $\mathrm{NIH}$ grants R01MH085542, R01MH093725, P50MH066392, P50MH080405, R01MH097276, RO1-MH-075916, P50M096891, P50MH084053S1, R37MH057881, AG02219, AG05138, MH06692, R01MH110921, R01MH109677, R01MH109897, U01MH103392, and contract HHSN271201300031C through IRP NIMH. Brain tissue for the CommonMindConsortium was obtained from the following brain bank collections: the Mount Sinai NIH Brain and Tissue Repository, the University of Pennsylvania Alzheimer's Disease Core Center, the University of Pittsburgh NeuroBioBank and Brain and Tissue Repositories, and the NIMH Human Brain Collection Core. CMC Leadership: Panos Roussos, Joseph Buxbaum, Andrew Chess, Schahram Akbarian, Vahram Haroutunian (Icahn School of Medicine at Mount Sinai), Bernie Devlin, David Lewis (University of Pittsburgh), Raquel Gur, Chang-Gyu Hahn (University of Pennsylvania), Enrico Domenici (University of Trento), Mette A. Peters, Solveig Sieberts (Sage Bionetworks), Thomas Lehner, Stefano Marenco, Barbara K. Lipska (NIMH).

\section{AUTHOR CONTRIBUTIONS}

This study was conceived and designed by GH, SB, NES, OAA. Data was acquired by $\mathrm{SB}, \mathrm{KOC}, \mathrm{OF}, \mathrm{AS}, \mathrm{FB}, \mathrm{CCF}, \mathrm{OBS}, \mathrm{AMD}, \mathrm{SD}$ and $\mathrm{OAA}$. Analysis and interpretation of data was conducted by GH, SB, NES, OF, FB, CCF, OBS, SD, AMD and OAA. Drafting of the manuscript was conducted by GH. Critical revision was provided by GH, SB, NES, KOC, OF, AS, FB, LR, CCF, OBS, AMD, SD and OAA.

\section{COMPETING INTERESTS}

OAA has received speaker's honorarium from Lundbeck and is a consultant for Healthlytix. AMD is a founder of and holds equity interest in CorTechs Labs and serves on its scientific advisory board. He is also a member of the Scientific Advisory Board of Healthlytix and receives research funding from General Electric Healthcare (GEHC). The terms of these arrangements have been reviewed and approved by the University of California, San Diego in accordance with its conflict of interest policies. Remaining authors have no conflicts of interest to declare.

\section{ADDITIONAL INFORMATION}

Supplementary information The online version contains supplementary material available at https://doi.org/10.1038/s41398-021-01576-4

Correspondence and requests for materials should be addressed to Guy Hindley or Ole A. Andreassen.

Reprints and permission information is available at http://www.nature.com/ reprints

Publisher's note Springer Nature remains neutral with regard to jurisdictional claims in published maps and institutional affiliations.

(i) Open Access This article is licensed under a Creative Commons Attribution 4.0 International License, which permits use, sharing, adaptation, distribution and reproduction in any medium or format, as long as you give appropriate credit to the original author(s) and the source, provide a link to the Creative Commons license, and indicate if changes were made. The images or other third party material in this article are included in the article's Creative Commons license, unless indicated otherwise in a credit line to the material. If material is not included in the article's Creative Commons license and your intended use is not permitted by statutory regulation or exceeds the permitted use, you will need to obtain permission directly from the copyright holder. To view a copy of this license, visit http://creativecommons. org/licenses/by/4.0/.

(c) The Author(s) 2021 